\title{
CERTAIN APPLICATIONS OF THE THEORY OF POLAR-COMPOSITE POLYNOMIALS
}

\author{
NEYAMAT ZAHEER AND MAHFOOZ ALAM
}

\begin{abstract}
In a recent paper [5] the authors have, for the first time, given a detailed account of the theory of polar-composite polynomials in algebraically closed fields of characteristic zero. In another paper [6], we have given some applications of this theory and have obtained a few results for a new variety of composite polynomials which have been derived from certain polar-composite polynomials through iteration. In the present paper also we consider the same variety of composite polynomials, but our present study deals with a different aspect of application of the said theory. Besides other things, our main theorem here offers a generalization of a result due to Marden [2] (see also [1, Theorem $(16,3)])$.
\end{abstract}

1. Introduction. The notations and the terminology in the present text is borrowed from [6] (refer also to [5] for fuller details). We shall denote by $D\left(\mathbf{C}_{\omega}\right)$ the class of all generalized circular regions of $\mathbf{C}_{\omega}$, where $\mathbf{C}_{\omega}$ represents the projective field of the field $\mathbf{C}$ of complex numbers found by adjoining to $\mathbf{C}$, the scalar infinity $\omega$. It is known (see [7, pp. 386-387, 352; 3, pp. 23, 35; or 4, p. 116]) that the nontrivial generalized circular regions of $\mathbf{C}_{\omega}$ are the open interior (or exterior) of circles or the half-planes, with a connected subset (possibly empty) of their boundary adjoined. We denote by $\mathcal{F}_{n}(\mathbf{C})$ the class of all $n$ th-degree polynomials from $\mathbf{C}$ to $\mathbf{C}$ and by $Z(f)$ the set of all zeros of the polynomial $f$.

The following lemma, due to the authors [6, Lemma (2.1)], will play a significant role in the proof of our main theorem in the next section.

LEMMA (1.1). Given $f \in \mathcal{F}_{m}(\mathbf{C}), \beta_{1} \in \mathbf{C}-\{0\}$ and an integer $p(1 \leq p \leq m)$, let us define

$$
h_{1}(z)=\beta_{1} f(z)+(-1)^{p} z^{p} f^{(p)}(z) \quad \text { for all } z \in \mathbf{C} .
$$

If $\beta_{1} \neq \lambda^{p}$, where $\lambda=-(p ! C(m, p))^{1 / p} \exp (i \pi / p)$, and if $A \in D\left(\mathbf{C}_{\omega}\right)$ such that $Z(f) \subseteq A$, then every zero $\gamma$ of $h_{1}(z)$ can be written in the form $\gamma=\alpha$ or $\gamma=$ $\alpha\left[\beta_{1 j} /\left(\beta_{1 j}-\lambda\right)\right]$ for some $\alpha \in A$ and for some member $\beta_{1 j}$ for some $j$ (the $\beta_{1 j}$, $0 \leq j \leq p-1$, being the pth-roots of $\left.\beta_{1}\right)$.

2. Main theorem. In this section we consider the same class of composite polynomials $h(z)$ as was considered earlier (see [6, Theorem (2.2)]), but this time we use a different aspect of application of Lemma (1.1) in proving our main theorem of this paper. This theorem deals with the general problem of locating the zeros of the composite polynomials $h(z)$ referred above, but in a different manner, so as to include in it a result due to Marden (see $[1$, Theorem $(16,3)$ or 2]). It may be

Received by the editors January 22, 1981 and, in revised form, August 10, 1981.

1980 Mathematics Subject Classification. Primary 30C10, Secondary 30C15.

Key words and phrases. Generalized circular regions, polar-composite polynomials.

(c) 1982 American Mathematical Society 0002-9939/82/0000-0216/\$02.25 
pointed out that the proof of our main theorem here employs similar techniques as those of an earlier theorem due to the authors (see [6, Theorem (2.2)]) except that, while applying Lemma (1.1) at the proper stage in the proof, we utilize arg $\gamma$ instead of $|\gamma|$.

THEOREM (2.1). Given the polynomials

$$
f(z)=\sum_{k=0}^{m} a_{k} z^{k}, \quad g(z)=\sum_{k=0}^{n} b_{k} z^{k} \quad\left(a_{m}, b_{n} \neq 0\right)
$$

from $\mathbf{C}$ to $\mathbf{C}$, let us form the polynomial

$$
h(z)=\sum_{k=0}^{m} a_{k} g\left((-1)^{p-1} p ! C(k, p)\right) z^{k}, \quad 1 \leq p \leq m .
$$

If all the zeros of $f(z)$ lie in the sector

$$
S_{0}: \mu_{1} \leq \arg z \leq \mu_{2}, \quad \mu_{2}-\mu_{1}=\mu<\pi,
$$

and if the pth-roots of all the zeros of $g(z)$ lie in the lune

$$
L: \theta_{1} \leq \arg [z /(z-\lambda)] \leq \theta_{2}, \quad\left|\theta_{1}\right|+\left|\theta_{2}\right| \leq(\pi-\mu) / n,
$$

where $\lambda=-(p ! C(m, p))^{1 / p} \exp (i \pi / p)$, then all the zeros of $h(z)$ lie in the sector

$$
S_{n}: \mu_{1}+\min \left(0, n \theta_{1}\right) \leq \arg z \leq \mu_{2}+\max \left(0, n \theta_{2}\right) \text {. }
$$

ProOF. Let $\beta_{1}, \beta_{2}, \ldots, \beta_{n}$ be the zeros of $g(z)$. For each value of $k=1,2, \ldots, n$, if $\beta_{k j}\left(0 \leq j \leq p_{1}\right)$ denotes the $p$ th-roots of $\beta_{k}$, the hypothesis on the zeros of $g(z)$ implies that

$$
\theta_{1} \leq \arg \left[\beta_{k j} \backslash\left(\beta_{k j}-\lambda\right)\right] \leq \theta_{2}, \quad\left|\theta_{1}\right|+\left|\theta_{2}\right| \leq(\pi-\mu) / n
$$

for all values of $k$ and $j$ and, hence that $\beta_{k j} \neq 0, \lambda$ for any values of $k$ or $j$. That is,

$$
\beta_{k} \neq 0, \lambda^{p} \quad \forall k=1,2, \ldots, n .
$$

If $\left\{g_{k}(z)\right\}$ and $\left\{h_{k}(z)\right\}$ are sequences of polynomials defined by

$$
\begin{gathered}
g_{k}(z)=b_{n}\left(\beta_{1}-z\right)\left(\beta_{2}-z\right)\left(\beta_{2}-z\right) \cdots\left(\beta_{k}-z\right), \\
h_{0}(z)=f(z), \quad h_{k}(z)=\beta_{k} h_{k-1}(z)+(-1)^{p} z^{p} h_{k-1}^{(p)}(z)
\end{gathered}
$$

for $k=1,2, \ldots, n$, then we know (see [6, proof of Theorem (2.2)]) that

$$
h_{k} \mathcal{F}_{m}(\mathbf{C}) \quad \forall k=1,2, \ldots, n
$$

and that

$$
h_{n}(z)=(-1)^{n} b_{n}^{-1} h(z), \quad g_{n}(z)=(-1)^{n} g(z),
$$

whatever originally be the value of $n$. To prove the theorem, it remains only to show that

$$
Z\left(h_{n}\right) \subseteq S_{n}
$$

To this effect we consider the sector $S_{k}(k=1,2, \ldots, n)$ given by

$$
S_{k}: \mu_{1}+\min \left(0, k \theta_{1}\right) \leq \arg z \leq \mu_{2}+\max \left(0, k \theta_{2}\right) \text {. }
$$


If we write

$$
A_{1}=\left\{z: \mu_{1} \leq \arg z \leq \mu_{1}+\pi\right\}, \quad A_{2}=\left\{z: \mu_{2}-\pi \leq \arg z \leq \mu_{2}\right\},
$$

then $A_{1}, A_{2} \in D\left(\mathbf{C}_{\omega}\right)$ such that $Z(f) \subseteq S_{0}=A_{1} \cap A_{2}$, because $\mu_{2}-\mu_{1}=\mu<\pi$. In view of this fact and the relation (2.2), we see that Lemma (1.1) is applicable to the polynomial $h_{1}(z)$ in each of the cases when the set $A_{1}$ or $A_{2}$ is substituted for the set $A$ in the lemma. Therefore, for each value of $i=1,2$, every zero $\gamma$ of $h_{1}(z)$ can be written in the form $\gamma=\alpha$ or $\gamma=\alpha\left[\beta_{1 j} /\left(\beta_{1 j}-\lambda\right)\right]$ for some $\alpha \in A_{i}$ and for some $\beta_{1 j}$ for some $j$. Now, the definitions of the sets $A_{i}$ and the inequalities (2.1) help us to conclude that $\arg \gamma$ must satisfy one of the inequalities

$$
\mu_{1} \leq \arg \gamma \leq \mu_{1}+\pi \text { or } \mu_{1}+\theta_{1} \leq \arg \gamma \leq \mu_{1}+\pi+\theta_{2}
$$

as well as one of the inequalities

$$
\mu_{2}-\pi \leq \arg \gamma \leq \mu_{2} \quad \text { or } \quad \mu_{2}-\pi+\theta_{1} \leq \arg \gamma \leq \mu_{2}+\theta_{2} .
$$

From this it follows that $\arg \gamma$ satisfies the inequalities

$$
\mu_{1}+\min \left(0, \theta_{1}\right) \leq \arg \gamma \leq \mu_{1}+\pi+\max \left(0, \theta_{2}\right)
$$

and

$$
\mu_{2}-\pi+\min \left(0, \theta_{1}\right) \leq \arg \gamma \leq \mu_{2}+\max \left(0, \theta_{2}\right) .
$$

Since $\mu_{2}-\mu_{1}<\pi$ and since

$$
\mu_{1}+\min \left(0, \theta_{1}\right) \leq \mu_{1} \leq \mu_{2} \leq \mu_{2}+\max \left(0, \theta_{2}\right)
$$

we have that

$$
\mu_{1}+\min \left(0, \theta_{1}\right) \leq \arg \gamma \leq \mu_{2}+\max \left(0, \theta_{2}\right) \text {. }
$$

That is, (2.4) holds true for $n=1$. Now assume that (2.4) holds for $n=k-1$. Then $h_{k-1} \in \mathcal{F}_{m}(\mathbf{C})$, due to (2.3), and all the zeros of the polynomial $h_{k-1}$ lie in the sector

$$
S_{0}^{\prime}: \mu_{1}^{\prime} \leq \arg z \leq \mu_{2}^{\prime}
$$

where

$$
\mu_{1}^{\prime}=\mu_{1}+\min \left(0,(k-1) \theta_{1}\right), \quad \mu_{2}^{\prime}=\mu_{2}+\max \left(0,(k-1) \theta_{2}\right) .
$$

Since (2.2) holds, the definition of $h_{k}$ and the statement (2.4) for $n=1$ (the case already proved), with $h_{1}$ replaced by $h_{k-1}$ and $\beta_{1}$ by $\beta_{k}$, imply that $Z\left(h_{k}\right) \subseteq S_{1}^{\prime}$, where

$$
S_{1}^{\prime}: \mu_{1}^{\prime}+\min \left(0, \theta_{1}\right) \leq \arg z \leq \mu_{2}^{\prime}+\max \left(0, \theta_{2}\right) .
$$

Finally the relations

$$
\mu_{1}^{\prime}+\min \left(0, \theta_{1}\right)=\mu_{1}+\min \left(0, k \theta_{1}\right)
$$

and

$$
\mu_{2}^{\prime}+\max \left(0, \theta_{2}\right)=\mu_{2}+\max \left(0, k \theta_{2}\right)
$$

imply that $Z\left(h_{k}\right) \subseteq S_{k}$ and (2.4) holds true for $n=k$. Therefore, (2.4) has been established for all $n$ by mathematical induction. This completes the proof.

The following corollary is a result due to Marden [1, Theorem $(16,3)]$ (see also [2, Theorem II]). 
COROLlARY (2.2). With the notation of Theorem (2.1), if $Z(f) \subseteq S_{0}$ and $Z(g) \subseteq L_{0}$, where

$$
L_{0}=\left\{z \in \mathbf{C}: \theta_{1} \leq \arg [z /(z-m)] \leq \theta_{2}\right\}, \quad\left|\theta_{1}\right|+\left|\theta_{2}\right| \leq(\pi-\mu) / n,
$$

then all the zeros of the polynomial

$$
H(z)=\sum_{k=0}^{m} a_{k} g(k) z^{k}
$$

lie in the sector $S_{n}$.

Proof. In Theorem (2.1) if we take $p=1$, then we notice that $\lambda=m, L=L_{0}$ and that $h(z)=H(z)$. Our corollary is now immediate from Theorem (2.1).

Next, we give a new result as application of the main theorem.

THEOREM (2.3). If all the zeros of the polynomial $f(z)=\sum_{k=0}^{m} a_{k} z^{k}$ are positive and real and if $p$ is an integer, $1 \leq p \leq m$, then all the zeros of the polynomial

$$
f_{p \beta}(z)=\sum_{k=0}^{m}\left\{(-1)^{p-1} k(k-1) \cdots(k-p+1)-\beta\right\} a_{k} z^{k}
$$

can be made to lie in any convex sector $S: \theta_{1} \leq \arg z \leq \theta_{2}$ containing the positive real axis in its interior, provided

$$
|\beta|>p ! C(m, p) \operatorname{cosec}^{p} \theta, \quad \text { where } \theta=\min \left(\left|\theta_{1}\right|,\left|\theta_{2}\right|\right) .
$$

ProOF. If $S$ is any sector as stipulated in the theorem, then $-\pi<\theta_{1}<0<$ $\theta_{2}<\pi$ and $\theta_{2}-\theta_{1}=\left|\theta_{1}\right|+\left|\theta_{2}\right| \leq \pi$. For each value of $i=1,2$, the arc of circle given by

$$
C_{i}=\left\{z \in \mathbf{C}: \arg [z /(z-\lambda)]=\theta_{i}\right\}
$$

is the locus of points at which the line segment from $z=0$ to $z=\lambda$ subtends a constant angle $\theta_{i}$ (see Theorem (2.1) for the value of $\lambda$ ). Then $C_{1}$ and $C_{2}$ are the arcs of circles, having end points at $z=0$ and $z=\lambda$ and lying on opposite sides of the above line segment, with diameters given by $d_{1}=|\lambda| \operatorname{cosec}\left|\theta_{1}\right|$ and $d_{2}=|\lambda| \operatorname{cosec}\left|\theta_{2}\right|$, respectively. These expressions hold even if $\left|\theta_{1}\right|$ or $\left|\theta_{2}\right|$ exceeds $\pi / 2$. Obviously, for each value of $i=1,2$, every point $z \in C_{i}$ satisfies the inequality $|z| \leq d_{i}$ or $|z| \leq|\lambda|$ according as $\left|\theta_{i}\right| \leq \pi / 2$ or $\left|\theta_{i}\right|>\pi / 2$. Since $\left|\theta_{1}\right|$ and $\left|\theta_{2}\right|$ cannot exceed $\pi / 2$ simultaneously and since $|\lambda| \leq d_{i}=|\lambda| \operatorname{cosec}\left|\theta_{i}\right|$ for $i=1,2$, all the points $z \in C_{1} \cup C_{2}$ satisfy the inequality $|z| \leq d_{1}$ or $|z| \leq d_{2}$ according as $\left|\theta_{2}\right|>\pi / 2$ or $\left|\theta_{1}\right|>\pi / 2$. That is, in either case when $\left|\theta_{1}\right|$ or $\left|\theta_{2}\right|$ exceeds $\pi / 2$, we have

$$
|z| \leq|\lambda| \operatorname{cosec} \theta \quad \forall z \in C_{1} \cup C_{2},
$$

where $\theta=\min \left(\left|\theta_{1}\right|,\left|\theta_{2}\right|\right)$. Obviously, (2.6) still holds when both $\left|\theta_{1}\right|$ and $\left|\theta_{2}\right|$ do not exceed $\pi / 2$. To sum up: In all cases, the inequality (2.6) holds for all points in the closed interior of the region bounded by $C_{1}$ and $C_{2}$.

Now if we take $g(z)=z-\beta$, with $\beta$ satisfying the inequality (2.5), then all the $p$ th-roots of $\beta$ (the only zero of $g(z)$ ) lie on the circle $|z|=|\beta|^{1 / p}$, where $|\beta|^{1 / p}>$ $|\lambda| \operatorname{cosec} \theta$. But the set of all points lying in the closed exterior of the region bounded by $C_{1}$ and $C_{2}$ (except the points 0 and $\lambda$ ) is precisely the lune $L$ given by

$$
L: \theta_{1} \leq \arg [z /(z-\lambda)] \leq \theta_{2}, \quad\left|\theta_{1}\right|+\left|\theta_{2}\right| \leq \pi .
$$


Therefore, all the $p$ th-roots of the zero of $g(z)$ lie in the lune $L$. Since all the zeros of $f(z)$ lie in the sector $S_{0}$ given by $S_{0}: 0 \leq \arg z \leq 0$, the polynomials $f$ and $g$ satisfy the hypotheses of Theorem (2.1) with $\mu_{1}=\mu_{2}=\mu=0$ and $n=1$. Consequently (since $\theta_{1}<0<\theta_{2}$ ), all the zeros of the polynomial $h(z)$ of Theorem (2.1), with $g(z)=z-\beta$, lie in the sector $S: \theta_{1} \leq \arg z \leq \theta_{2}$. Finally, it is easy to see that the polynomial $f_{p \beta}(z)$ of the present theorem is precisely the above polynomial $h(z)$. The proof is now complete.

REMARK (2.4). (I) Since the polynomial $-f_{p \beta}(z)$ in Theorem (2.3) turns out, incidently, to be the polynomial $h_{1}(z)$ of Lemma (1.1) with $\beta_{1}=\beta$, the above theorem can also be proved by using, basically, the same method of proof as above and applying Lemma (1.1) instead of Theorem (2.1).

(II) If $0<\alpha<\pi$, Theorem (2.3) says that all the zeros of $f_{p \beta}(z)$ lie in the sector

$$
H_{\alpha}: \alpha-\pi \leq \arg z \leq \alpha,
$$

provided $|\beta|>p ! C(m, p) \operatorname{cosec}^{p} \gamma$, where $\gamma=\min (|\alpha-\pi|,|\alpha|)$. If, in particular, $\alpha=\pi / 2$, then $H_{\alpha}=\{z:-\pi / 2 \leq \arg z \leq \pi / 2\}$ and all the zeros of $f_{p \beta}(z)$ lie in the half-plane $\operatorname{Re}(z) \geq 0$, provided $|\beta|>p ! C(m, p)$.

COROLLARY (2.5). Under the notations and hypotheses of Theorem (2.3) no zero of $f_{p \beta}(z)$ can be a nonpositive real number for any value of $\beta$ such that

$$
|\beta|>p ! C(m, p) \text {. }
$$

ProOF. Since $a_{0}=f(0) \neq 0$, we see that $f_{p \beta}(0)=-\beta f(0) \neq 0$ for any $\beta$ satisfying (2.7). It remains only to show that no zero of $f_{p \beta}(z)$ can be a negative real number for any $\beta$ satisfying (2.7). In order to prove this we suppose, on the contrary, that $f_{p \beta}(\lambda)=0$ for some negative real number $\lambda$ and for some $\beta$ satisfying (2.7). From Remark (2.4)(II) we, therefore, conclude that $\lambda=\operatorname{Re}(\lambda) \geq 0$. Since $\lambda \neq 0$ (already proved), we must have $\lambda>0$. This contradicts the choice of $\lambda$ already made, and our corollary is established.

COROLLARY (2.6). If all the zeros of an mth-degree polynomial $f(z)$ are real and positive and if $p$ is an integer, $1 \leq p \leq m$, then

$$
|z|^{p}\left|f^{(p)}(z)\right| \leq p ! C(m, p)|f(z)| \quad \forall z \in H,
$$

where $H=\{z \in \mathbf{C}: \operatorname{Re}(z)<0\}$.

ProOF. If we suppose, on the contrary, that

$$
\left|z_{0}\right|^{p}\left|f^{(p)}\left(z_{0}\right)\right|>p ! C(m, p)\left|f\left(z_{0}\right)\right| \text { for some } z_{0} \in H,
$$

then (since $f\left(z_{0}\right) \neq 0$ ) we must have

$$
\left|(-1)^{p-1} z_{0}^{p} f^{(p)}\left(z_{0}\right) / f\left(z_{0}\right)\right|>p ! C(m, p) .
$$

Putting $\beta=(-1)^{p-1} z_{0}^{p} f^{(p)}\left(z_{0}\right) / f\left(z_{0}\right)$, we observe that $|\beta|>p ! C(m, p)$ and that

$$
0=\beta f\left(z_{0}\right)+(-1)^{p} z_{0}^{p} f^{(p)}\left(z_{0}\right)=-f_{p \beta}\left(z_{0}\right)
$$

as stated in Remark (2.4)(I). Now Remark (2.4)(II) implies that $\operatorname{Re}\left(z_{0}\right) \geq 0$, contradicting the fact that $z_{0} \in H$. The corollary is now proved.

At the end, we prove the following result on the roots of certain partial fraction sums as application of Theorem (2.3). 
COROllaRY (2.7). If, for $j=1,2, \ldots, q$, the $t_{j}$ are positive reals and the $m_{j}$ are positive integers with sum $m$, then the roots of the equation

$$
\sum_{j=1}^{q} m_{j} /\left(z-t_{j}\right)=\beta / z
$$

for all complex numbers $\beta$ lying outside the circle $|z|=m$, lie in the closed half-plane $\operatorname{Re}(z) \geq 0$.

ProOF. If we put

$$
f(z)=\prod_{j=1}^{q}\left(z-t_{j}\right)^{m_{j}}=\sum_{k=0}^{m} a_{k} z^{k}
$$

and take $\beta \in \mathbf{C}$ such that $|\beta|>m$, then the polynomial $f_{p \beta}(z)$ of Theorem (2.3) for $p=1$ is given by

$$
-f_{1 \beta}(z)=\sum_{k=0}^{m}(\beta-k) a_{k} z^{k}=\beta f(z)-z f^{\prime}(z) .
$$

Since the polynomial $f$ satisfies the hypotheses of Theorem (2.3), with $p=1$ and $-\theta_{1}=\theta_{2}=\pi / 2$, we conclude (see also Remark (2.4)(II)) that all the zeros of $f_{1 \beta}(z)$ lie in the closed half-plane $\operatorname{Re}(z) \geq 0$ (since $|\beta|>m$ ). Since the left-hand side of equation (2.9) equals $f^{\prime}(z) / f(z)$, every root of equation (2.9) must be a zero of $f_{1 \beta}(z)$. Hence, the corollary has been established.

\section{REFERENCES}

1. M. Marden, Geometry of polynomials, 2nd ed., Math. Surveys, no. 3, Amer. Math. Soc., Providence, R. I., 1966. MR 37 \#1562.

2. - The zeros of certain composite polynomials, Bull. Amer. Math. Soc. 49 (1943), 93-100.

3. N. Zaheer, Null-sets of abstract homogeneous polynomials in vector spaces, Doctoral thesis, Univ. of Wisconsin, Milwaukee, 1971.

4. - On polar relations of abstract homogeneous polynomials, Trans. Amer. Math. Soc. 218 (1976), 115-131.

5. N. Zaheer and M. Alam, Zeros of polar-composite polynomials in algebraically closed fields, Proc. London Math. Soc. (3) 40 (1980), 527-552.

6. - Some applications of the theory of polar-composite polynomials, J. London Math. Soc. (2) 22 (1980), 403-410.

7. S. P. Zervos, Aspects modernes de la localisation des zéros des polynômes d'une variable, Ann. Sci. École Norm. Sup. (3) 77 (1960), 303-410. MR 23 \#A3241.

Department of Mathematics, Aligarh MUSlim University, Aligarh-202001, INDIA (Current address of Mahfooz Alam)

Current address (Neyamat Zaheer): Department of Mathematics, Faculty of Science, King Saud University, Riyadh, P. O. Box 2455, Kingdom of Saudi Arabia 